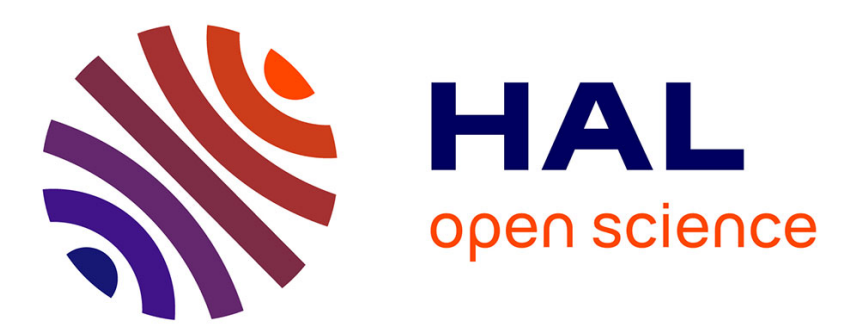

\title{
Mesures hydrologiques par profileur à effet Doppler (aDcp) en France: application aux cours d'eau et aux réseaux urbains
}

J. Le Coz, F. Larrarte, G. Saysset, G. Pierrefeu, J.F. Brochot, P. Marchand

\section{- To cite this version:}

J. Le Coz, F. Larrarte, G. Saysset, G. Pierrefeu, J.F. Brochot, et al.. Mesures hydrologiques par profileur à effet Doppler (aDcp) en France: application aux cours d'eau et aux réseaux urbains. La Houille Blanche - Revue internationale de l'eau, 2009, 3, p. 115 - p. 122. hal-00547581

\section{HAL Id: hal-00547581 \\ https://hal.science/hal-00547581}

Submitted on 16 Dec 2010

HAL is a multi-disciplinary open access archive for the deposit and dissemination of scientific research documents, whether they are published or not. The documents may come from teaching and research institutions in France or abroad, or from public or private research centers.
L'archive ouverte pluridisciplinaire HAL, est destinée au dépôt et à la diffusion de documents scientifiques de niveau recherche, publiés ou non, émanant des établissements d'enseignement et de recherche français ou étrangers, des laboratoires publics ou privés. 


\title{
Mesures hydrologiques par profileur à effet Doppler (aDcp) en France : application aux cours d'eau et aux réseaux urbains
}

\author{
Hydrological measurements by Doppler profiling (aDcp) in France : \\ application to streams and urban networks
}

JEROME LE COZ

Cemagref, Unité de Recherche Hydrologie-Hydraulique, 3bis quai Chauveau - CP 220, 69336 Lyon cedex 9, France tel. +33 (0)4 722087 86, e-mail : jerome.lecoz@cemagref.fr

FREDERIQUE LARRARTE

LCPC, Division Eau et Environnement, BP 4129, 44341 Bouguenais cedex, France

\section{GERARD SAYSSET}

EDF-Division Technique Générale, 18 avenue Poincaré, BP 423, 19311 Brive cedex, France

\section{GILLES PIERREFEU}

Compagnie Nationale du Rhône, Laboratoire Hydraulique et Mesures, 4 rue de Châlon-sur-Saône, 69007 Lyon, France

\author{
JEAN-FRANÇOIS BROCHOT \\ DIREN Rhône-Alpes SHAC, BP 606, 21016 Dijon, France
}

\section{PIERRE MARCHAND}

IRD Martinique, 3 rue de la rose des vents BP 8006, 97259 Fort-de-France cedex, France

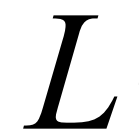

es profileurs acoustiques de vitesse par effet Doppler (aDcp, pour " acoustic Doppler current profiler ») sont de plus en plus utilisés pour le jaugeage des cours d'eau en France. Après un bref rappel du principe et de la mise en cuvre de l'aDcp, les actions du groupe d'utilisateurs français (Groupe Doppler) sont présentées, en particulier concernant les incertitudes de mesure, le problème du fond mobile et le cas des profileurs fixes horizontaux (H-aDcp). Des essais prometteurs menés en réseaux d'assainissement urbains sont également décrits.

coustic Doppler current profilers (aDcp) are increasingly used for gauging streams in France. After an overview of the principle of operation and deployment techniques, the efforts of the French user group (Groupe Doppler) are presented, in particular as regards measurement uncertainty, mobile-bed and fixed horizontal profilers (H-aDcp). Promising tests conducted in urban drainage networks are also reported.

\section{I INTRODUCTION}

Les profileurs acoustiques de vitesse par effet Doppler (aDcp, pour « acoustic Doppler current profiler ») sont de plus en plus utilisés pour le jaugeage des cours d'eau, notamment en France depuis 1994. Ces appareils apparaissent plus sophistiqués et plus complexes à maîtriser que les techniques conventionnelles d'exploration des vitesses (moulinets, courantomètres électromagnétiques, etc.), mais le calcul du débit repose sur des principes similaires. Cet article présente le principe de la mesure de débit par aDcp et les questions soulevées par son application aux cours d'eau et aux réseaux urbains. Le retour d'expérience des utilisateurs français, au sein du Groupe Doppler, est mis en perspective avec les travaux sur la qualité des débits.

\section{ACTIONS CONCERTÉES SUR LE JAUGEAGE PAR ADCP EN FRANCE}

\footnotetext{
II.1 MESURES DE DÉBIT PAR ADCP

La mesure de vitesse par l'aDcp repose sur le principe du Doppler pulsé : émission d'impulsions ultrasonores dans l'eau et analyse du décalage fréquentiel de l'écho rétrodiffusé par les particules en suspension. Les aDcp commercialisés comportent généralement 3 ou 4 transducteurs émettant des faisceaux acoustiques divergents autour de la verticale, ce qui permet de mesurer le profil vertical des vitesses 3D (Fig. 1-a).
} 
L'intégration des vitesses normales obtenues sur une section de rivière permet d'obtenir le débit. Le plus souvent, l'appareil est capable de se positionner lui-même en mesurant la vitesse apparente du fond à l'aide d'impulsions spécifiques (suivi de fond / bottom-tracking). On parle alors de jaugeage mobile autonome, par opposition au déploiement non autonome, i.e. couplé à un système de positionnement externe (GPS). L'acquisition de profils verticaux sur des positions fixes (mode stationnaire ou « verticale par verticale ») permet également d'obtenir le débit sans recours au suivi de fond, ni au GPS.

Dans tous les cas, les vitesses de certaines zones de la section ne peuvent pas être mesurées en raison de limitations techniques (Fig. 1-b). Ainsi les débits doivent être extrapolés dans des portions limitées en haut et bas de section, ainsi qu'aux bords (par manque de profondeur).

Les modes de déploiement vont de systèmes de fixation à une embarcation (Fig. 2-a) aux supports flottants (planche, mini-catamaran ou trimaran ) tracté ou radiocommandé (Fig. 2-b). Quels que soient le support et la façon de le déplacer, les manœuvres doivent être menées le plus souplement et lentement possible, en respectant une période initiale de mise en température. La limite d'utilisation de l'aDcp est surtout la vitesse en surface (sécurité des personnels, remous nuisibles à la mesure). Des vitesses supérieures à $4,5 \mathrm{~m} / \mathrm{s}$, voire à $5 \mathrm{~m} / \mathrm{s}$ ont toutefois pu être mesurées sur l'Ain et sur l'Ardèche par exemple. L'utilisation de l'aDcp peut également être inadaptée en présence de faibles tirants d'eau, de surface libre agitée, de bulles, etc. L'hydromètre doit alors lui préférer une méthode de jaugeage plus appropriée (courantomètres, dilution).

\section{II.2 LE GROUPE DOPPLER}

Le Groupe Doppler est né du besoin ressenti par de nombreux utilisateurs français d'aDcp de partager leurs expériences, exprimer leurs besoins méthodologiques/métrologiques et organiser des travaux concertés. Constitué au tout début de 2005 à l'initiative de la CNR, d'EDF et du Ministère en charge de l'Environnement, le groupe est indépendant de tout constructeur ou fournisseur et ouvert à tout utilisateur désireux d'échanger sur les applications de l'aDcp, en particulier le jaugeage des cours d'eau. La communication au sein du groupe est facilitée par une liste de diffusion
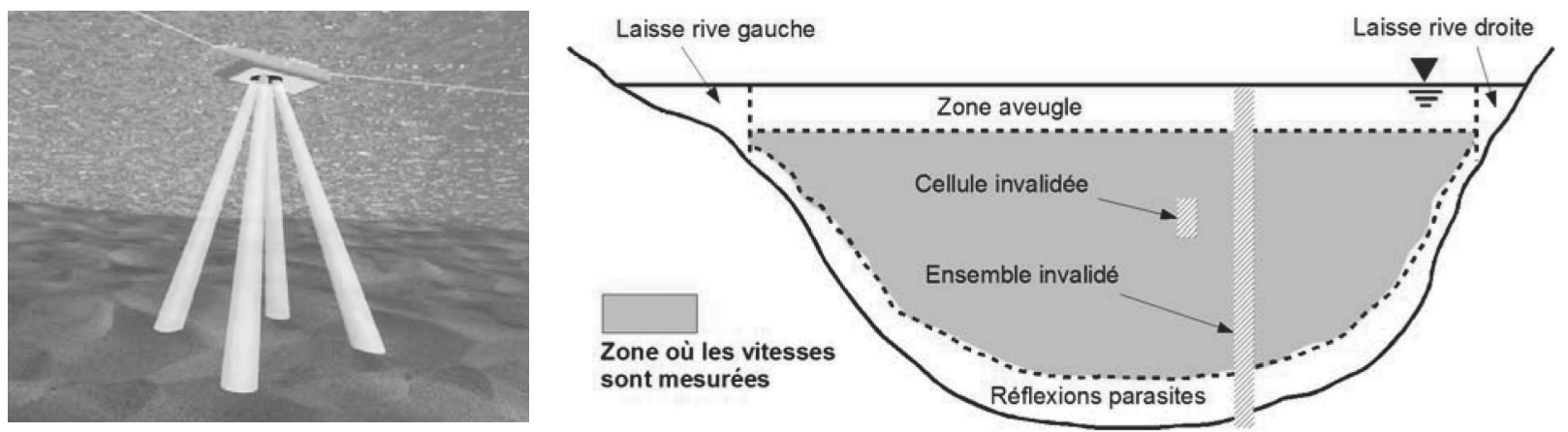

Fig. 1 - a) Configuration « Janus » d'un aDcp à quatre faisceaux ;

b) Zones de la section où les débits sont mesurés et zones où ils doivent être extrapolés.
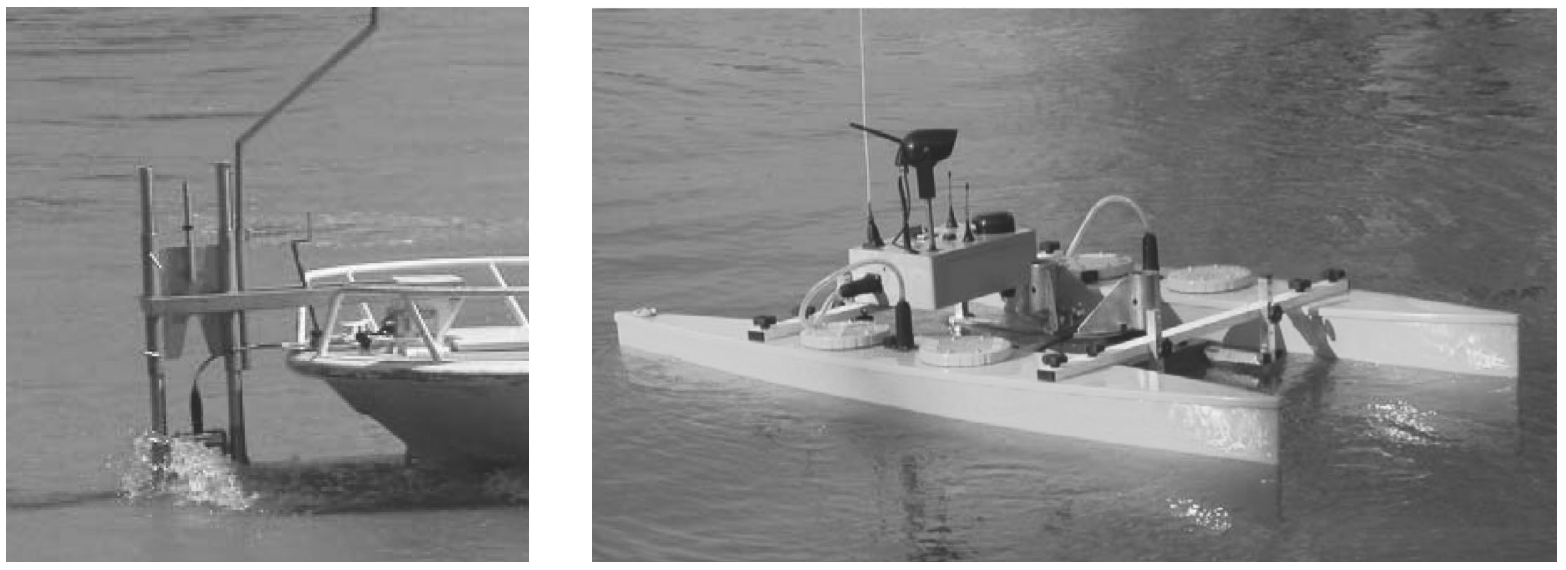

Fig. 2 - a) Montage sur potence à l'avant d'une vedette ; b) Mini-catamaran radio-commandé 
électronique SYMPA (avec archivage des échanges et des documents) hébergée par le serveur informatique du Cemagref à Lyon (une centaine d'adresses électroniques abonnées au 01/03/2009). Une équipe restreinte assure la coordination des travaux du groupe. Des réunions plénières ont lieu chaque année.

La plupart des utilisateurs opérationnels emploient l'aDcp en routine sur les réseaux hydrométriques dont ils ont la charge. Les équipes qui manient l'appareil depuis longtemps tendent à privilégier son usage, même si les autres techniques (courantomètres mécaniques, électromagnétiques, ou à effet Doppler, dilution) continuent d'être employées, notamment pour contrôler les mesures par aDcp. Le jaugeage par aDcp reste toutefois inadapté voire impossible sur de nombreuses sections (surface trop agitée par exemple) et pour des crues violentes (corps flottants, mise en danger des opérateurs). La gamme de sections jaugées est très étendue, depuis des largeurs de $10 \mathrm{~m}$ et des profondeurs de quelques dizaines de centimètres à des largeurs de plusieurs kilomètres et des profondeurs de l'ordre de $50 \mathrm{~m}$ (Amazone, IRD). Des vitesses moyennes extrêmement faibles (quelques $\mathrm{cm} / \mathrm{s}$ ) ont $\mathrm{pu}$ être mesurées de façon satisfaisante, la limite supérieure se situant aux alentours de $4 \mathrm{~m} / \mathrm{s}$, essentiellement à cause des contraintes de déploiement et de sécurité.

La plupart des profileurs utilisés par les membres du Groupe Doppler sont de marque Teledyne RDI : ADCP ${ }^{\circledR}$ « classiques » (BroadBand 300, 600 ou $1200 \mathrm{kHz}$, WorkHorse 600 ou $1200 \mathrm{kHz}$ ), StreamPro® $2400 \mathrm{kHz}$ pour faibles tirants d'eau $(<4 \mathrm{~m})$. Sont également employés des aDcp de marque SonTek/YSI (mini-ADP ${ }^{\circledR}$ et ADP $®$ ), LinkQuest (FlowQuest ${ }^{\circledR}$ ) et NorTek/OTT-Qmetrix (Q-Liner $\left.{ }^{\circledR}\right)$.

\section{II.3 MÉTHODOLOGIE, FORMATION, INTERNATIONAL}

Le Groupe Doppler a pour objectif de contribuer à la réflexion méthodologique sur les protocoles de mesure et d'exploitation des mesures de débit par aDcp. Ceci s'est traduit par une synthèse bibliographique de la littérature scientifique et technique internationale [1], la définition d'une fiche jaugeage type, et la rédaction d'un guide pratique « Mesures hydrologiques par profileur Doppler $(\mathrm{aDcp}) »[2]$, publié aux Editions QUAE (Cemagref-Cirad-Ifremer-INRA) en 2008.

Une première partie présente les principes de fonctionnement de l'appareil et de son application au jaugeage des cours d'eau (calcul du débit). Une deuxième partie concerne le jaugeage proprement dit : le matériel et son déploiement ; paramétrage des aDcp TRDI ; protocole de jaugeage ; établissement et critique du débit jaugé. Enfin, des compléments sont apportés sur les autres utilisations de l'aDcp en rivière (bathymétrie, vitesses, transport sédimentaire, débitmétrie en continu).

Sur la base de ce document, des actions de formation ont été menées dans le cadre de la formation continue des agents d'EDF-DTG ainsi que des services de l'Etat (IFORE).

Les membres du Groupe Doppler participent régulièrement à des conférences, comme par exemple les colloques «ADCPs in Action » organisés par Teledyne RDI, le congrès international « Experiences and Advancements in Hydrometry » à Séoul en mars 2008, ou encore le colloque SHF " Mesures hydrologiques et incertitudes ». Ces conférences permettent de faire connaître les pratiques et les travaux des utilisateurs français d'aDcp, et en retour de se tenir informé des démarches similaires à l'étranger.

Au niveau international, le Groupe Doppler est représenté au sein du groupe de travail international sur les instruments et techniques de mesure de débit que l'OMM (Organisation Météorologique Mondiale - Genève) a constitué en 2007 [3]. L'équipe coordinatrice comprenant des représentants de différentes organisations (AIRH, AISH, ISO, HMEI, OMM) se réunit depuis 2007 lors de conférences téléphoniques ou de séminaires comme en 2008 à Séoul [4]. Le groupe OMM prévoit la réalisation d'un recensement des techniques et instruments de débitmétrie, ainsi que des normes/procédures nationales/internationales. En outre, il proposera un cadre pour l'établissement des incertitudes et la conduite des tests métrologiques. La documentation et les rapports de tests collectés seront mis à disposition sur un site web public (http:// www.wmo.int/pages/prog/hwrp/FlowMeasurement.html).

Enfin, un groupe technique ISO travaille à l'élaboration d'une norme, dans le prolongement de la spécification technique ISO/TS 24154 [5]. Le Groupe Doppler français a proposé de contribuer à cette réflexion.

\section{II.4 RÉGATES ET TESTS EN COMMUN}

Une régate $\mathrm{aDcp}$ est un test métrologique in situ consistant à jauger simultanément la même section avec plusieurs aDcp. Une telle opération permet de vérifier le bon accord des différents appareils entre eux, et éventuellement l'impact du type de déploiement, du paramétrage choisi, etc. La seule comparaison des aDcp entre eux ne permet pas de détecter un éventuel biais collectif, ni de départager deux groupes de mesure discordantes. Il est par conséquent toujours utile de réaliser la régate en présence d'une mesure de débit indépendante, considérée comme référence, pour des conditions hydrauliques stables et prédictibles dans la mesure du possible.

L'agence de l'Environnement britannique teste chacun de ses appareils de cette manière au moins tous les deux ans [6]. En France, des régates aDcp ont notamment été organisées en interne par la CNR et EDF-DTG. L'expérience des régates passées montre que la comparaison d'aDcp entre eux fournit normalement des résultats identiques à moins de $2 \%$ près s'ils sont utilisés dans le même mode. Toutefois, des différences significatives ont parfois pu être décelées, pour des paramétrages différents.

Le Groupe Doppler s'est proposé d'organiser des régates aDcp multi-organismes, pour différents types de section, allant du canal usinier du Rhône au torrent de montagne, en passant peut-être par un faciès intermédiaire. Les 3-4-5 février 2009, une première régate $\mathrm{aDcp}$ organisée sur la Vézère au pont de Garavet, à Allassac en Corrèze a permis de comparer les débits mesurés par 35 appareils déployés par 21 équipes, soit 7 modèles d'ADCP commercialisés par 4 constructeurs concurrents et émettant des ultrasons à des fréquences comprises entre 600 et $3000 \mathrm{kHz}$ selon les appareils [7]. Pour un débit constant pendant les séries 
de mesure, les jaugeages ont été effectués simultanément sur 11 sections parallèles espacées de $18 \mathrm{~m}$ en amont du pont, plus 1 section correspondant à un déploiement depuis le pont. Les supports flottants permettant de déployer les appareils ont été très variés : bras métallique depuis une embarcation légère, mini-catamaran ou mini-trimaran, radiocommandé ou tracté manuellement grâce à une corde tendue en travers de la rivière.

Les débits fournis par les différents appareils ont été comparés entre eux, ainsi qu'aux débits de référence issus de la station hydrométrique du Saillant. Le troisième jour, la DTG de Brive a également pratiqué un jaugeage par dilution chimique (Rhodamine WT). En moyenne tous les débits obtenus sont en très bon accord entre eux (écarts de $5 \%$ ou moins). Des écarts ponctuels plus importants ont été observés, et leur cause sera analysée pour savoir s'il s'agit d'appareils défectueux ou d'erreurs dues à un paramétrage ou à un protocole inadaptés. Ces très bons résultats sont à replacer dans le contexte de mesure très favorable (site, régime hydraulique constant, météo radieuse), et ne doivent pas être extrapolés à tout jaugeage ADCP.

Cette première régate $\mathrm{ADCP}$ inter-organisme française a été appréciée par l'ensemble des participants, qui ont pu également bénéficier d'échanges techniques avec d'autres hydromètres parfois éloignés géographiquement, dans une ambiance très conviviale. Le Groupe Doppler envisage de réitérer ce type d'essais, sur d'autres types de cours d'eau, plus torrentiels, ou plus grands comme le Rhône par exemple (déploiement par bateau à moteur).

\section{Q QUALITÉ DE LA MESURE DE DÉBIT}

Cette section rassemble trois des principaux thèmes d'actualité pour le Groupe Doppler concernant la qualité de la mesure de débit par aDcp et $\mathrm{H}-\mathrm{aDcp}$. De plus amples détails sont fournis dans le guide pratique [2].

\section{III.1 CONTRÔLES IN-SITU, INCERTITUDES}

La plupart des jaugeurs ont cherché à évaluer expérimentalement la qualité des jaugeages par aDcp par comparaison avec d'autres techniques mieux connues. Les écarts rapportés dans la littérature sont quasi-systématiquement inférieurs à $5 \%$, dans de bonnes conditions d'application. A titre d'exemple, la CNR a effectué 21 jaugeages aDcp en autocontrôle de 1995 à 2004 sur 14 sites différents, pour une gamme de débits comprise entre 0 et $3500 \mathrm{~m}^{3} / \mathrm{s}$ [8]. Dans $80 \%$ des cas l'écart avec le jaugeage par moulinet est inférieur à $4 \%$, et dans $20 \%$ des cas entre 4 et $10 \%$ à cause d'instabilités hydrauliques. Une analyse métrologique permet d'associer à un jaugeage aDcp réalisé dans de bonnes conditions une incertitude proche de $5 \%$ [9].

Par ailleurs, l'USGS a analysé un jeu de 1032 transects $\mathrm{aDcp}$, correspondant à 100 débits de référence (moulinet, courbe de tarage, station à ultrasons), pour des largeurs de chenal de 7 à $500 \mathrm{~m}$ et des profondeurs de 0,5 à $9 \mathrm{~m}$ [10]. Quel que soit le mode d'acquisition, les débits obtenus par aDcp apparaissent non biaisés, et pour une moyenne de qua- tre transects, l'incertitude communément admise de $5 \%$ est confirmée. Il ressort que la durée du jaugeage est plus importante que le nombre de traversées moyennées pour réduire cette incertitude. Les deux paramètres étant en pratique liés, le Groupe Doppler préconise la réalisation d'au moins 6 à 8 traversées successives, sous conditions hydrauliques stationnaires.

La formalisation du calcul des incertitudes liées à la mesure de débit par aDcp reste délicate et fait l'objet de travaux de recherche finalisée [11]. En lien étroit avec les opérationnels membres du Groupe Doppler, ainsi que l'université d'Iowa et l'USGS, un post-doctorat Cemagref pour le développement, l'implémentation et l'évaluation d'un outil de calcul des incertitudes associées à un jaugeage aDcp est programmé pour 2009-2011. Dans cette optique, des tests en laboratoire sont utiles pour quantifier les incertitudes élémentaires associées aux mesures brutes (vitesses de l'eau et $\mathrm{du}$ fond notamment).

\section{III.2 DÉTECTION ET CORRECTION DU FOND MOBILE}

En mode mobile autonome (référence « bottom-track »), l'aDcp fonde son calcul de débit sur l'hypothèse d'un fond immobile afin de pouvoir se repérer dans l'espace sans accessoire complémentaire comme le GPS. En présence d'un transport de fond actif, le fond se déplace vers l'aval, les vitesses et donc le débit brut fourni par l'aDcp sont systématiquement sous-estimés. Virtuellement, la trajectoire du bateau vue par l'aDcp aura tendance à remonter vers l'amont. Lors d'un aller-retour entre deux positions fixes de part et d'autre de la section, la dérive de trajectoire cumulée permet de quantifier la vitesse apparente moyenne du fond, et donc de corriger la sous-estimation du débit.

L'exemple fourni Fig. 3- $a$ est instructif, puisqu'en présence d'un fond mobile, la CNR a déployé deux aDcp RioGrande 600 et $1200 \mathrm{kHz}$, ce dernier étant en outre couplé à un GPS différentiel centimétrique. Le débit brut fourni par le $1200 \mathrm{kHz}$ en mode autonome $\left(8400 \mathrm{~m}^{3} / \mathrm{s}\right)$ est significativement inférieur à celui fourni par le $600 \mathrm{kHz}$ en mode autonome $\left(8900 \mathrm{~m}^{3} / \mathrm{s}\right)$, lui-même très proche de celui fourni par le $1200 \mathrm{kHz}$ en référence GPS $\left(8950 \mathrm{~m}^{3} / \mathrm{s}\right)$, mode de calcul insensible au fond mobile. En toute logique, les deux débits en mode autonome ne peuvent être que sous-estimés, d'autant plus que la fréquence des ultrasons est élevée (plus faible pénétration dans le substrat). Considérant simplement le biais global de $9,8 \mathrm{~cm} / \mathrm{s}$ sur la vitesse de fond, le débit à $1200 \mathrm{kHz}$ corrigé est ramené à une valeur proche des deux autres $\left(8850 \mathrm{~m}^{3} / \mathrm{s}\right)$

\section{III.3 LES ADCP FIXES HORIZONTAUX (H-ADCP)}

Depuis 2005, plusieurs aDcp fixes à visée horizontale (H-aDcp) ont été installés en France : équipement du Rhône puis de la Saône à Lyon par la CNR [12], équipement de l'Isère à Romans avec plusieurs $\mathrm{H}-\mathrm{aDcp}$ par la CNR, équipement du Rhône à proximité des centrales nucléaires de St-Alban et Tricastin par EDF-DTG [13], et sur le canal usinier de Montélimar par la CNR. Fonctionnant sur le même 
principe que les aDcp classiques, les H-aDcp offrent une mesure continue (moyenne multipings sur 1 minute typiquement) des vitesses horizontales sur une ligne horizontale à cote fixe, en travers de la section.

Deux types de méthodes permettent alors de calculer le débit total à partir des vitesses mesurées [14]. Soit les potentiels unitaires sont calculés à l'aide d'une bathymétrie et d'une loi théorique des profils de vitesse pré-établies (cas des logiciels actuellement utilisés sur les stations EDF et CNR). Soit une corrélation est établie entre la vitesse débitante obtenue par jaugeage et une vitesse représentative calculée à partir des mesures de l'aDcp-H (méthode « index velocity » utilisée notamment par l'USGS).

A Saint-Georges, où la section est scrutée sur quasiment toute la largeur, les écarts en débit avec 18 jaugeages aDcp de 100 à $1800 \mathrm{~m}^{3} / \mathrm{s}$ réalisés en 2006 étaient inférieurs à $5 \%$, sauf quelques jaugeages à bas débit sous-estimés. Toutefois, pour d'autres installations (CNR/EDF) en 2006-2007 sur le Rhône ou en canal usinier, pour des sections scrutées sur une partie seulement de la largeur, les écarts observés étaient plutôt de l'ordre de 10 à $20 \%$ avec une sous-estimation systématique, et avec parfois des instabilités. Des problèmes de mesure des vitesses, notamment en présence d'écoulements complexes, et en situation de faibles vitesses et faibles concentrations en MES, ont été mis en évidence [15]. La portée exploitable de l'appareil est alors drastiquement réduite, voire nulle. Une analyse fine des paramètres vitesse/écho apparaît pour exploiter cette technologie.

Eu égard au fort potentiel de tels appareils, le Groupe Doppler va poursuivre un effort d'analyse des problèmes rencontrés (en lien avec le constructeur) et des stratégies de calcul des débits. En outre, une thèse est menée par le Cemagref sur la mesure en continue par aDcp-H des flux d'eau et de matières en suspension, ce qui pose le problème

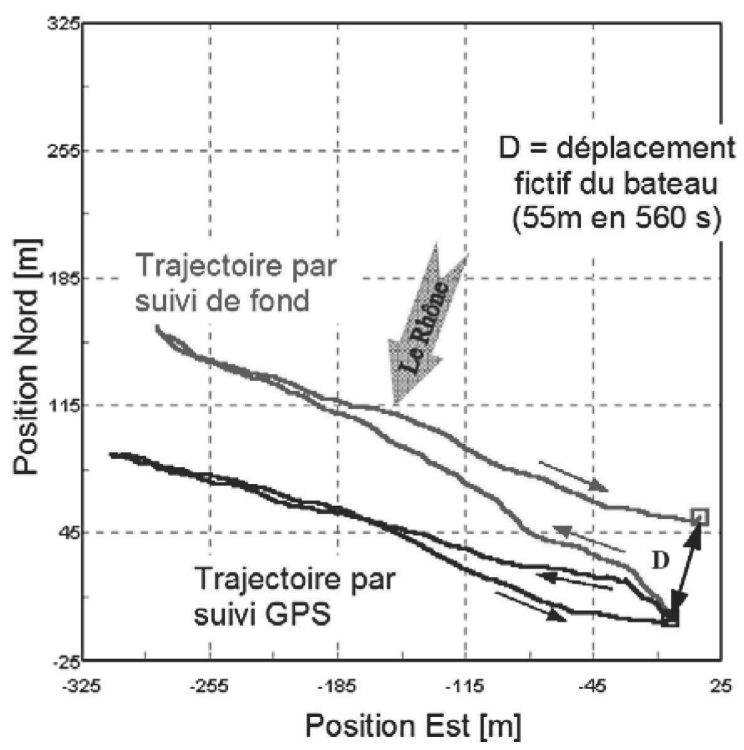

de la conversion de l'intensité acoustique rétrodiffusée en concentration.

\section{IV a APPLICATIONS EN RÉSEAUX URBAINS}

\section{IV.1 RAPPEL DU CONTEXTE}

Les réseaux d'assainissement urbains s'équipent progressivement de points de mesure en continu des débits, avec des objectifs d'évaluation des performances et/ou de gestion en temps réel. Pour ce faire, la mesure de hauteur est souvent complétée par une mesure de vitesse, visant à obtenir la vitesse moyenne dans la section de mesure. Plusieurs technologies sont utilisées. La plus couramment employée est celle du Doppler « continu ». La mise en œuvre d'une mesure de vitesse en continu ne dispense pas totalement de la réalisation de jaugeages de contrôle : les capteurs ne mesurent la vitesse que dans un volume réduit et son extrapolation à l'ensemble de la section est soumise à des hypothèses sur le champ de vitesse. Dans ce contexte, le GEMCEA (Groupement pour l'Evaluation des Mesures en Continu En Assainissement) et le LCPC (Laboratoire Central des Ponts et Chaussées) ont lancé un programme de recherche sur la qualification des sites de mesure et la conception de leur instrumentation. Ce projet comporte une étude détaillée des champs de vitesse et de leur sensibilité à l'influence des singularités telles que des coudes [16] et comprend une partie expérimentale pour laquelle du matériel spécifique a été développé afin d'effectuer des relevés de vitesses [17]. Ce matériel a également permis de mettre en œuvre un profileur Doppler pulsé, et de comparer les résultats obtenus avec d'autres capteurs.

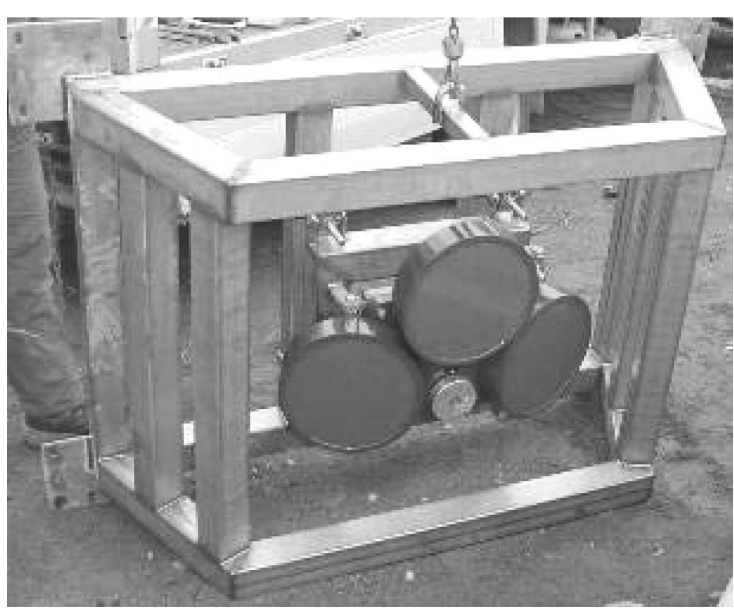

Fig. 3 - a) Exemple de suivi de fond faussé par fond mobile (Rhône à Beaucaire le 27/11/2002) ; b) H-aDcp $300 \mathrm{kHz}$ de la station CNR de St-Georges (Saône à Lyon). 


\section{IV.2 MONTAGE EXPÉRIMENTAL}

Le capteur utilisé est un PC-ADP (Pulse Coherent Acoustic Doppler Profiler) fabriqué par la firme américaine SonTek. Il présente trois transducteurs, qui sont des émetteurs-récepteurs d'ultrasons à $1500 \mathrm{kHz}$. Ils sont orientés tous les $120^{\circ}$ en plan horizontal et inclinés de $15^{\circ}$ par rapport à l'axe vertical. La tête est fixée de telle sorte que l'un des faisceaux est face à l'écoulement. Le PC-ADP est relié à un boîtier convertissant le signal émis par le profilographe en données binaires transmises dans notre cas vers un ordinateur. Le matériel acquis est une évolution " shallow water » (faibles hauteurs d'eau) du modèle usuellement proposé par le fabricant. Cela se traduit par une bande morte («blank ») de $0,10 \mathrm{~m}$ et un maximum de 100 cellules, chaque cellule ayant une hauteur de $0,02 \mathrm{~m}$. Les mesures ont été faites avec un pas de temps de $10 \mathrm{~s}$.

Après différents essais de prise en main, le PC-ADP a été fixé sur un dispositif d'exploration des vitesses dans la section appelé Cerbère [17] dont on utilise ici le degré de liberté vertical pour maintenir le PC-ADP au contact de la surface libre (Fig. 4-a), car en collecteur la hauteur d'eau est variable selon l'heure de la journée et les événements pluvieux.

\section{IV.3 EXEMPLES DE RÉSULTATS}

Le PC-ADP a attiré notre attention dans le cadre de l'étude des distributions spatiales des vitesses dans un collecteur. En effet, conçu pour être fixé à la surface de l'écoulement, il doit permettre i) d'acquérir des informations sur un profil vertical en un temps très bref ; ii) d'obtenir des mesures près $\mathrm{du}$ fond. Le profileur PC-ADP est un appareil qui permet d'acquérir de nombreuses données mais ses dimensions et son poids rendent sa mise en œuvre complexe. A contrario cet appareil est un quasi-prototype acquis dans une optique de recherche et non a des fins de gestion ou de suivi en continu, il n'a donc fait l'objet d'aucune démarche de miniaturisation ou de portabilité.

$\mathrm{Du}$ fait de l'inclinaison de $15^{\circ}$ des transducteurs avec la verticale, chacun des trois volumes de mesure correspondant à la cellule i située à la profondeur $d_{i}$ se trouve à une distance horizontale $\Delta=d_{i} \tan \left(15^{\circ}\right)$ de l'axe vertical du profileur. Par exemple, $\Delta=0,52 \mathrm{~m}$ à $1 \mathrm{~m}$ de profondeur. Les vitesses indiquées par le PC-ADP pour chaque cellule sont donc calculées à partir des vitesses radiales mesurées à l'intérieur de trois volumes dont l'écartement croît avec la profondeur. Compte tenu des dimensions du collecteur, les fais-
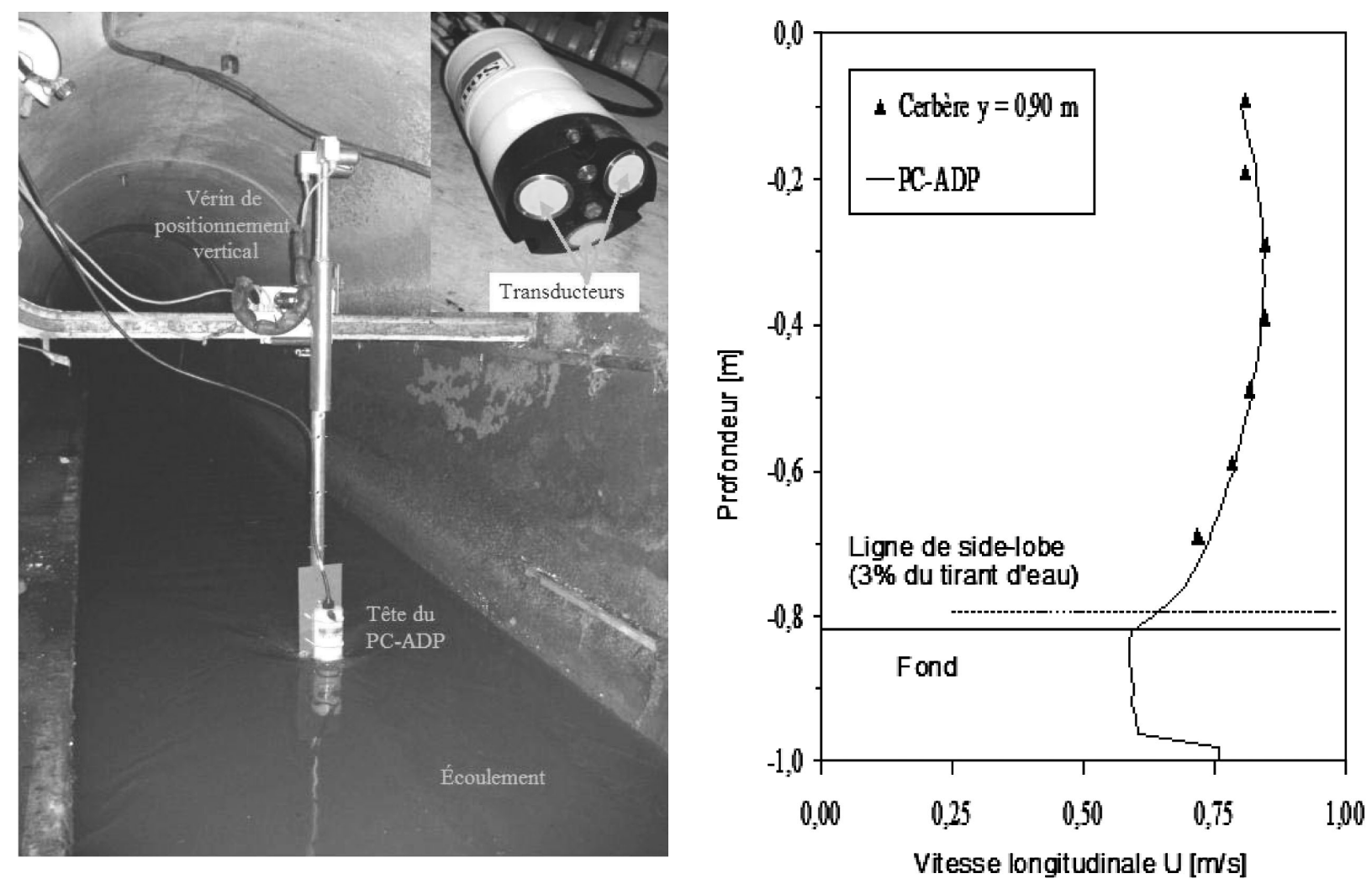

Fig. 4 - a) Profileur PC-ADP fixé au montage Cerbère et détail de la tête ; b) Comparaison des mesures PC-ADP avec les données Cerbère. 
ceaux couvrent une large fraction transversale de la section de mesure. Les profils des vitesses transversales et verticales correspondent en effet à un étalement spatial pouvant représenter plus du quart de la section transversale. Le PC-ADP n'est donc pas un appareil adapté à l'étude des distributions des vitesses transversales et verticales dans la section.

Par contre, comme la section de mesure est uniforme sur $50 \mathrm{~m}$ en amont et $2 \mathrm{~m}$ en aval de la section dans laquelle est installée le PC-ADP, nous avons donc considéré que la vitesse longitudinale était bien représentative d'un profil vertical de vitesses. Nous avons essayé de travailler avec un seul faisceau ou en inclinant la tête pour disposer deux des faisceaux dans un plan vertical mais les résultats étaient incohérents, sans doute sous l'effet d'un prétraitement du signal par le logiciel d'acquisition. Nous avons donc comparé les profils verticaux de vitesse longitudinale donnés par le PC-ADP dans sa configuration normale avec ceux obtenus avec l'échantillonneur Cerbère [17] installé dans un collecteur du réseau d'assainissement nantais.

La Fig. 4-b montre un exemple de profil de vitesse longitudinale. L'ordonnée est ici la distance à la surface libre. On voit nettement un décrochage du profil de vitesses lorsque les ultrasons arrivent au niveau du béton constituant le radier du collecteur. Le PC-ADP ne permet pas de mesure immédiatement sous sa tête du fait de l'existence d'une zone morte mais permet par contre de scruter l'écoulement jusqu'à une distance réduite du radier. En raison des échos de lobes secondaires, cette distance peut être évaluée à $3 \%$ du tirant d'eau pour une ouverture des faisceaux de $15^{\circ}[1]$. Soit, pour notre appareil dont les cellules ont une épaisseur de $2 \mathrm{~cm}$, un maximum de 2 cellules pour 1,5 $\mathrm{m}$ de hauteur d'eau.

La Fig. 4-b montre que le profil donné par le PC-ADP est en très bon accord avec celui au milieu de la section transversale en $\mathrm{y}=0,90 \mathrm{~m}$. Une comparaison des profils obtenus pour des hauteurs d'eau de 0,6 à $0,8 \mathrm{~m}$ a montré que les écarts restent toujours inférieurs à $4 \%$. Cet essai a été renouvelé à plusieurs reprises et a donné des résultats identiques. Le PC-ADP est donc un outil permettant une bien meilleure détermination des profils au fond que les autres appareils actuellement disponibles.

\section{$V \square$ CONCLUSIONS}

Suite à la généralisation des mesures de débit par aDcp en France depuis une quinzaine d'années, les utilisateurs se sont organisés pour échanger leurs expériences, définir des pratiques communes et conjuguer leurs développements technologiques et métrologiques. Un guide méthodologique a été publié, des actions de communication scientifique et technique sont menées dans le cadre de congrès nationaux et internationaux, et de formation continue des équipes hydrométriques. Une première régate multi-organismes a été organisée et dépouillée en 2009, d'autres devraient suivre. Pour l'heure, trois objectifs de travail se distinguent nettement : 1) établir et éprouver une méthode de calcul des incertitudes associées à un débit mesuré par $\mathrm{aDcp}$; 2) continuer à formaliser des bonnes pratiques et des méthodes permettant de surmonter les limitations de la technique face aux difficultés du terrain, à l'exemple du travail réalisé sur le problème du fond mobile ; 3 ) étudier et améliorer la mesure de vitesse et le calcul du débit par les H-aDcp. Lors de tests en réseau urbain, l'aDcp a également montré son potentiel pour l'étude des profils verticaux de vitesse longitudinale, au moins au centre de l'écoulement et notamment près du fond. Cela ouvre des perspectives pour l'étude des phénomènes pariétaux et la prise en compte du transport solide. Les résultats présentés dans cet article illustrent la fécondité du partenariat entre chercheurs et opérationnels dans le cadre de programmes de recherche finalisée sur les techniques hydrométriques innovantes.

\section{DEMERCIEMENTS}

Les auteurs remercient l'ensemble des membres du Groupe Doppler pour leur contribution aux échanges et travaux, ainsi que les personnels du LCPC et de la Direction de l'Assainissement de la Communauté Urbaine de Nantes pour leur aide lors des tests en réseaux d'assainissement.

\section{VII — RÉFÉRENCES}

[1] Le Coz J., Chastan B., Vedie F., Dramais G. (2007) Emplois de l'aDcp en rivière : une revue de synthèse. $L a$ Houille Blanche. 04 119-130

[2] Le Coz J., Pierrefeu G., Saysset G., Brochot J.-F., MARCHAND P. (2008) - Mesures hydrologiques par profileur Doppler (aDcp) - Guide pratique, Editions QUAE. $192 \mathrm{p}$

[3] Fulford J. M., Pilon P., Kopaliani Z., Mccurry P., Caponi C. (2007) - Call for collaboration in WMO project for the assessment of the performance of flow measurement instruments and techniques. J. Hydraulic Eng. 133 (12) 1439-1440

[4] Le Coz J. (2008) - Challenges in Hydrometry : Some Examples from France. In International. Symposium "Experiences \& Advancements in Hydrometry", 18-20 mars 2008, Séoul, Corée du Sud.

[5] (2005) - Hydrometry - Measuring river velocity and discharge with acoustic Doppler profiler. Norme ISO/TS 24154 :2005. $10 \mathrm{p}$

[6] Iredale R. (2006) - A proposed method of assessing the comparative performance of ADCP's. Results from the Environment Agency "ADCP Regatta », River Severn, Bewdley. In RD Instruments ADCPs in Action - Europe, Cannes, France.

[7] Le Coz J., Saysset G., Pierrefeu G. (2009) - Régate ADCP 1, 3-4-5/02/2009, Vézère au pont de Garavet (Allassac, Corrèze), rapport technique (Groupe Doppler).

[8] Pierrefeu G. (2004) - Effects of a moving bottom on a discharge measurement. Discharge measurements using a DGPS. In RD Instruments ADCPs in Action - Europe, Nice, France.

[9] Olivier A., Blanquart B., Pierrefeu G., Scotti M. (2008) - Incertitude sur les mesures de débit issues d'un jaugeage à l'ADCP. Colloque SHF «Mesures hydrologiques et incertitudes », 1-2 avril 2008, Paris, France.

[10] Oberg K. A., Mueller D. S. (2007) - Validation of streamflow measurements made with Acoustic Doppler Current Profilers. J. Hydraulic Eng. 133 (12) 1421-1432 
[11] Gonzalez-Castro J. A., Muste M. (2007) - Framework for estimating uncertainty of ADCP measurements from a moving boat by standardized uncertainty analysis. J. Hydraulic Eng. 133 (12) 1390-1410

[12] Pierrefeu G. (2006) - Monitoring the Saône river discharge in Lyon by H-ADCP. In RD Instruments ADCPs in ActionEurope, Cannes, France.

[13] Legras D. (2006) — Setting up of a $300 \mathrm{kHz}$ narrowbeam H-ADCP upstream Saint Alban nuclear power plant on the Rhône river. In RD Instruments ADCPs in Action - Europe, Cannes, France.

[14] Hauet A., Le Coz J., Dramais G., Carre C., Legras D., Pierrefeu G., Godayer C. (2009) - Méthodes innovantes pour la mesure des débits fluviaux en continu : profileur
Doppler fixe horizontal (H-aDcp) et analyse d'images (LSPIV). Colloque SHF " Mesures hydrologiques et incertitudes 》, 1-2 avril 2008, Paris, France.

[15] Le Coz J., Pierrefeu G., Paquier A. (2008) - Evaluation of river discharges monitored by a fixed side-looking Doppler profiler (H-aDcp), doi : 10.1029/2008WR006967. Water Resources Research. 44, W00D09

[16] Bonakdari H., Larrarte F., Joannis C., Levacher D. (2007) - Une méthodologie d'aide à l'implantation de débitmètres en réseaux d'assainissement. $X X V^{\text {iemes }}$ Rencontres Universitaires de Génie Civil 2007 - Bordeaux 23-25 mai 2007, actes sur CD.

[17] LARRARTE F. (2006) — Velocity fields in sewers : an experimental study. Flow Measurement and Instrumentation. 17 (5) 282-290 